\title{
Diffuse progressive pulmonary interstitial and intra-alveolar cho- lesterol granulomas in childhood
}

\author{
K. Sato*, H. Takahashi*, H. Amano*, T. Uekusa**, T. Dambara*, S. Kira*
}

\begin{abstract}
Diffuse progressive pulmonary interstitial and intra-alveolar cholesterol granulomas in childhood. K. Sato, H. Takahashi, H. Amano, T. Uekusa, T. Dambara, S. Kira. (cERS Journals Ltd 1996.

ABSTRACT: We describe an 18 year old male with pulmonary interstitial and intra-alveolar cholesterol granulomas (PICG), which developed to severe respiratory failure over 15 yrs.

The histological diagnosis was made on the basis of open lung biopsy findings at the age of 3 yrs and autopsy at 18 yrs of age. Although the pathological features of the lung were similar to that of paediatric patients with lipoid pneumonia coexisting with pulmonary alveolar proteinosis (PAP), gastro-oesophageal reflux (GOR) and a diverse group of severe primary diseases, the patient lacked evidence for any of these.

We believe the present case provides a new example of a diffuse-type of lipoid pneumonia coexisting with pulmonary alveolar proteinosis, which we call cholesterol granulomas.
\end{abstract}

Eur Respir J., 1996, 9, 2419-2422.

\begin{abstract}
*Dept of Respiratory Medicine, Juntendo University School of Medicine, Tokyo, Japan. **Dept of Pathology, St Luke's International Hospital, Tokyo, Japan.

Correspondence: K. Sato, Dept of Respiratory Medicine, Juntendo University School of Medicine, 2-1-1 Hongo, Bunkyo-ku, Tokyo 113, Japan

Keywords: Diffuse interstitial shadows, endogenous lipoid pneumonia, pulmonary alveolar proteinosis, pulmonary interstitial and intra-alveolar, cholesterol granulomas, respiratory failure
\end{abstract}

Received: January 26 1996, Accepted after revision June 251996
Lipoid pneumonia is an uncommon condition resulting from the accumulation of lipid material from exogenous or endogenous sources in the lungs. Exogenous sources include mineral oil, administered as nose drops or laxatives, animal and vegetable oils [1-3]. Endogenous lipoid pneumonia (ELP) is usually associated with bronchial obstruction or, more rarely, certain chronic inflammatory diseases, such as bronchiectasis, lung abscess and tuberculosis [1]. Recently, diffuse ELP was described with gastro-oesophageal reflux (GOR) in paediatric patients with a diverse group of severe primary diseases [4].

We report the case of a patient with diffuse lipoid pneumonia of unknown aetiology, who developed respiratory failure over more than 15 yrs. Although aetiological antecedents were uncertain, a histological diagnosis was made after examining an open lung biopsy specimen taken at the age of 3 yrs and reconfirmed at autopsy at the age of $18 \mathrm{yrs}$. The histopathological findings were similar to those of ELP related to GOR, but differed in the predominant evidence of cholesterol accumulation. This case was also different from those reported previously, as the patient had neither GOR nor severe background diseases.

\section{Case Report}

The patient was born after an uncomplicated pregnancy and delivery and experienced no developmental or growth problems. His parents and brothers are fit and well and family history is unremarkable. At the age of $3 \mathrm{yrs}$, he visited another hospital for evaluation of fever and arthralgia, and a tentative diagnosis of juvenile rheu-matoid arthritis was made. Concurrently, abnormal shadows were evident on his chest radiograph, and open lung biopsy was performed to establish the diagnosis. The pulmonary histological appearance raised a strong suspicion of lipoid pneumonia with cholesterol clefts (fig. 1), although he had no history of aspiration or inhalation of mineral oil in the form of laxatives, nasal drops or throat sprays. Furthermore, he had no recognized episodes of GOR, or exposure to dust or inhaled irritant gas.

During his nursery and elementary schooling, he remained asymptomatic and was able to practise gymnastics. He began to experience dyspnoea on effort from 12 yrs of age, when he entered junior high school. Although dyspnoea progressed slowly, he was able to lead a normal daily life for the following 5 yrs. When

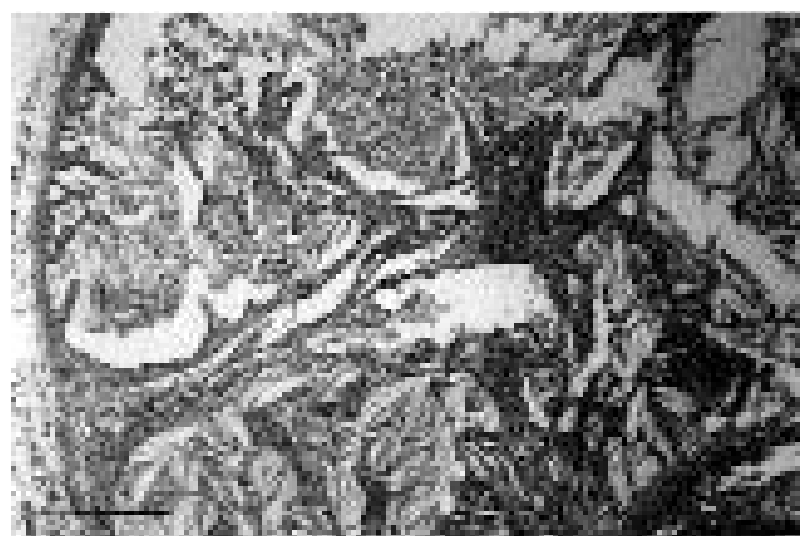

Fig. 1. - Microscopic appearance of the lung obtained by open lung biopsy at 3 yrs of age, showing severe widespread lipoid pneumonia with formation of prominent acicular cholesterol clefts. Alveolar wallthickening with lymphocytic infiltration is also observed (Haematoxylin and eosin stain; scale bar $500 \mu \mathrm{m}$ ). 
he was aged 17 yrs, he visited another hospital because severe dyspnoea had developed. A clinical diagnosis of pulmonary fibrosis was made based on chest radiographic findings of bilateral diffuse reticular shadows and severe hypoxaemia. As the hypoxaemia was severe and progressive, he was prescribed home oxygen therapy. Although the dyspnoea was alleviated by the oxygen therapy, his radiographic findings remained unchanged. His condition was stable for the next 4 months on home oxygen therapy. However, the dyspnoea then deteriorated further and he was referred to our hospital for further evaluation and treatment.

At the time of admission, he complained of severe dyspnoea, but denied having a cough, sputum or fever. Physical examination showed a severely emaciated male, whose height was $162 \mathrm{~cm}$ and weight $34 \mathrm{~kg}$. His temperature was $36.2^{\circ} \mathrm{C}$, pulse 108 beats $\cdot \mathrm{min}^{-1}$, with a regular rhythm, blood pressure $112 / 68 \mathrm{mmHg}$, and respiratory rate 50 breaths $\cdot \mathrm{min}^{-1}$. Prominent cyanosis with clubbed fingers, jugular venous dilatation and a pyramidal chest were observed, and fine crackles were audible in the bilateral lung fields. Other physical findings were unremarkable.

Laboratory data on admission were as follows: white blood cell count (WBC) $24.4 \times 10^{9}$ cells $\cdot \mathrm{L}^{-1}$ with neutrophilia and lymphocytosis; haemoglobin $15.1 \mathrm{~g} \cdot \mathrm{dL}^{-1}$; platelets $32.2 \times 10^{4} \cdot \mathrm{mm}^{-3}$; erythrocyte sedimentation rate (ESR) $27 \mathrm{~mm} \cdot \mathrm{h}^{-1}$; C-reactive protein (CRP) $9.9 \mathrm{mg} \cdot \mathrm{dL}^{-1}$; lactate dehydrogonase (LDH) $730 \mathrm{IU} \cdot \mathrm{L}^{-1}$; total cholesterol $150 \mathrm{mg} \cdot \mathrm{dL}^{-1}$; triglyceride $57 \mathrm{mg} \cdot \mathrm{dL}^{-1}$. Antinuclear and rheumatoid (RAPA) factors were slightly elevated, $80 \times$ and $40 \times$, respectively. Data from other routine laboratory tests, including liver and kidney function and immunoserological investigations, were within the normal ranges. Arterial blood gas analysis during nasal oxygen supplementation at a flow rate of $3 \mathrm{~L} \cdot \mathrm{min}^{-1}$, showed: $\mathrm{pH}$ 7.406; arterial oxygen tension $\left(\mathrm{Pa}_{\mathrm{a}} \mathrm{O}_{2}\right) 4.9 \mathrm{kPa}(37.0$ torr) and arterial carbon dioxide tension $\left(\mathrm{Pa}_{\mathrm{a}} \mathrm{CO}_{2}\right) 6.5 \mathrm{kPa}$ (48.4 torr). Electrocardiographic and echocardiographic findings suggested the presence of right heart overload. A chest radiograph on admission showed bilateral reticulonodular infiltrates, predominantly in the mid and lower lung fields (fig. 2), and a computed tomographic (CT) scan of the chest revealed diffuse interstitial infiltration accompanied by small alveolar filling shadows in both lungs (fig. 3). Cytological and bacteriological examinations of sputum could not be carried out, because the patient could not expectorate sputum. Pulmonary function tests, lung biopsy and bronchoalveolar lavage (BAL) could not be performed, because his respiratory distress was life-threatening.

After admission, the general condition of the patient was improved transiently by supportive therapy, including high-concentration oxygen inhalation with a reservoir bag and administration of low-dose oral prednisolone. However, the hypoxaemia gradually worsened with no evidence of respiratory tract infection, heart failure or pulmonary thromboembolism, and he died from respiratory failure 3 months after admission.

Postmortem examination showed stiff, indurated lungs (right $880 \mathrm{~g}$, left $680 \mathrm{~g}$; normal adult lung weight 625 and $565 \mathrm{~g}$, respectively) with no volume reduction. The surface of the lungs had a diffuse nodular appearance, and the cut surfaces showed diffuse fibrosis, honeycomb-like

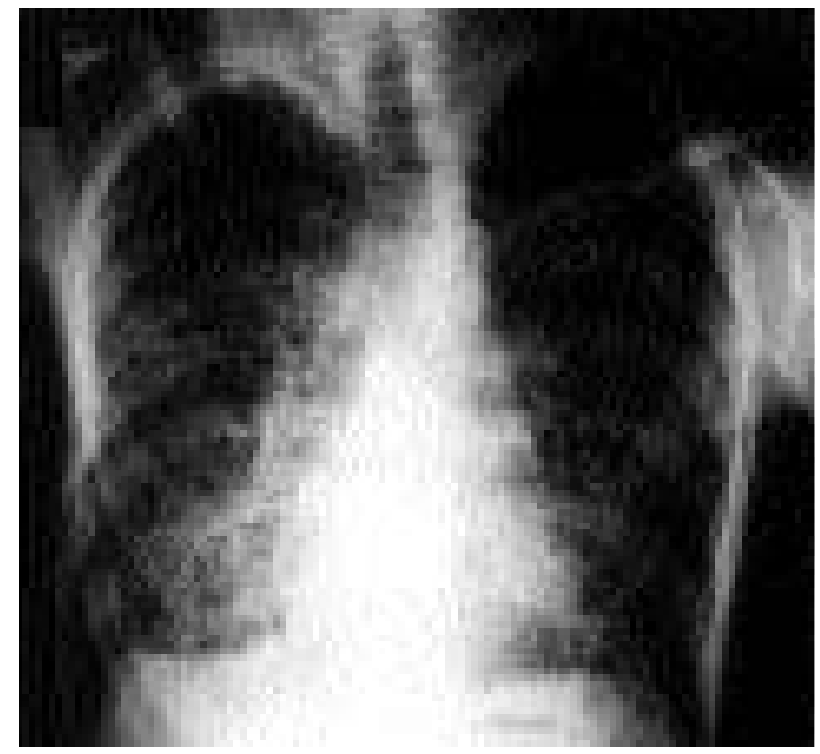

Fig. 2. - Posteroanterior chest radiograph on admission at 18 yrs of age showing diffuse reticulonodular infiltrates in the entire lung field.

appearance and numerous deposits of yellowish material, about 5-10 $\mathrm{mm}$ in diameter, bilaterally, and from the bottom to the top of each lung. Severe right ventricular wall hypertrophy and right ventricular dilatation reflecting cor pulmonale were also observed. Histopathological examination of the lung revealed severe, diffuse lipoid deposition in the alveolar and interstitial spaces, with pronounced proliferation of type II pneumocytes and an increased number of lipid-laden macrophages. Lipid stains with oil red $\mathrm{O}$ and nile blue sulphate showed red and violet, respectively, which suggested that the lipid deposition was of endogenous origin [2].

Other striking features were cholesterol clefts in the alveolar and interstitial spaces and alveolar wall-thickening with lymphocytic infiltrations (fig. 4a), which was called pulmonary interstitial and intra-alveolar cholesterol granulomas (PICG) [4]. These changes were evenly distributed throughout the entire lung and resulted in severe distortion of the native structure of the lung.

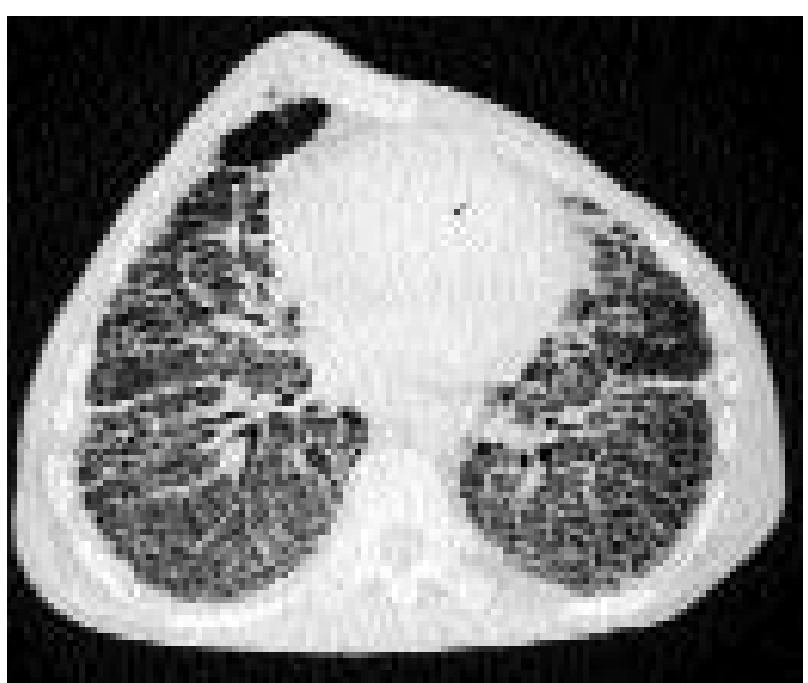

Fig. 3. - Computed tomography of the chest, showing diffuse, small reticular opacities and increase in alveolar and interstitial densities (window level -500 Hounsfield units (HU); window width 5,100 HU). 
a)

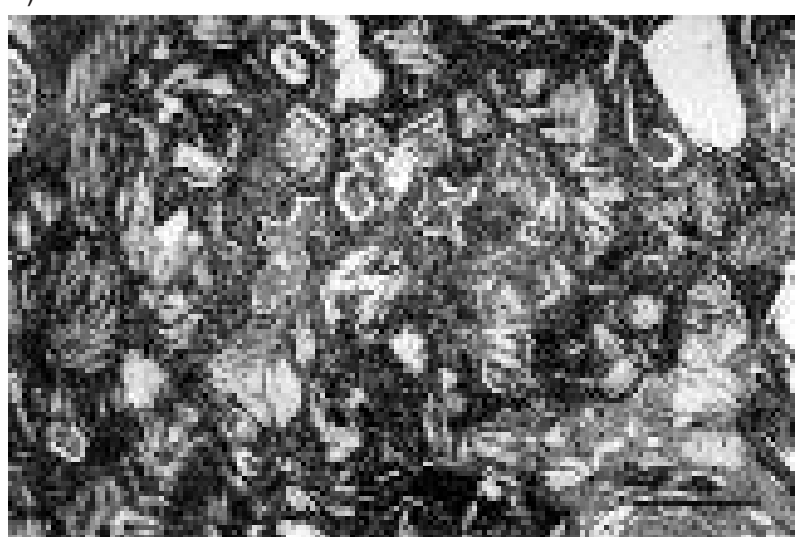

b)

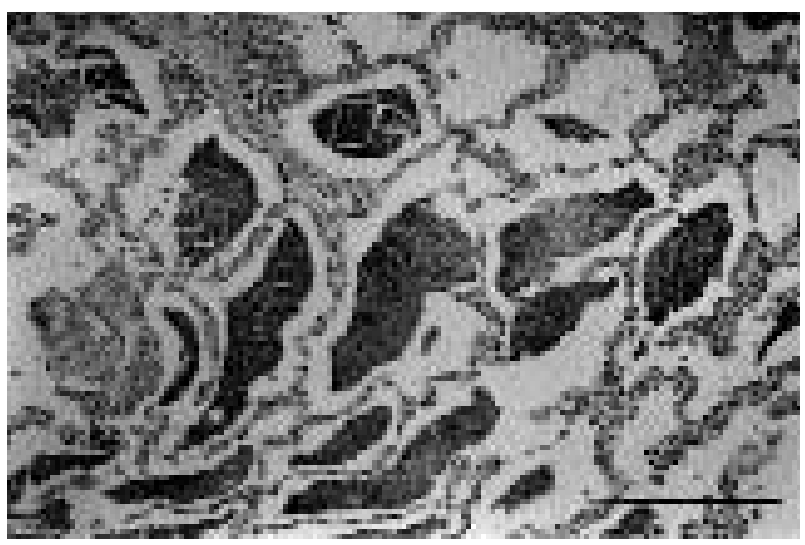

Fig. 4. - a) The microscopic appearance of the lung obtained at autopsy, showing foamy macrophages, alveolar wall-thickening with lymphocytic infiltrations and prominent acicular cholesterol clefts in alveolar and interstitial spaces (Haematoxylin and eosin stain; scale bar $500 \mu \mathrm{m})$. b) The microscopic appearance of another area of the lung, showing periodic-acid-Schiff (PAS)-positive acellular material in alveolar spaces (PAS stain; scale bar $300 \mu \mathrm{m}$ ).

Periodic-acid-schiff (PAS)-positive intra-alveolar acellular material was also readily apparent (fig. 4b). In general, this pulmonary alveolar proteinosis (PAP)-like change was much less extensive than the lipid deposition. Small arteries showed medial hypertrophy. No airway obstruction, foreign bodies, giant cells or granulomatous lesions were found. Electron microscopic investigation confirmed proliferation of type II pneumocytes, which contained electron-dense multilamellar bodies (data not shown). No abnormal lipid deposition was observed in any other organ, such as the liver, spleen or kidney, and no evidence of GOR, such as anatomical abnormalities of the gastro-oesophageal junction or lower oesophageal mucosal changes were found.

\section{Discussion}

The present case was unique because histological evidence of PICG and ELP was obtained at the age of 3 and $18 \mathrm{yrs}$, and the pathological features of the two specimens were very similar $[4,8,10]$. It is conceivable that the patient had had PICG and ELP for over $15 \mathrm{yrs}$ and it developed insidiously into chronic respiratory failure. Unlike the previously reported case of diffuse PICG and ELP, he did not have immunodeficiency state, heart failure or repetitive respiratory infection, which might have promoted development of respiratory failure. Therefore, this patient may represent a certain natural course of diffuse-type PICG and ELP.

Lipoid pneumonia is classified as exogenous or endogenous according to the source of lipid that accumulates in the lungs [1]. Although the most common sources of lipid within the alveoli, bronchioles and interstitial tissues are aspirated and/or inhaled exogenous mineral oils $[2,3]$, our patient had no evident history of exposure to mineral oils or other fat-like materials. Besides his clinical history, the diffuse distribution of the lesions in this case appeared to differ from that seen in exogenous lipoid pneumonia, which is usually predominant in the lower and right middle lobes [5-7]. Therefore, exogenous lipoid pneumonia was considered unlikely in this case.

It is known that PICG and ELP often arise when lung tissue breaks down distal to an obstructed airway, such as in lung cancer, lung abscess and bronchiectasis [1]. However, radiological and postmortem examinations showed no evidence of airway obstruction in the present case. Pulmonary hypertension was reported to be important in the pathogenesis of cholesterol granulomas in some cases [8]. Although right heart overload was apparent at autopsy in our patient, it appeared to be a change secondary to severe lung destruction and chronic respiratory failure due to the lipoid pneumonia. Other rare conditions, such as administration of cationic amphiphilic drugs (most commonly amiodarone), the cytotoxic drug busulphan and silicosis, have been reported to be responsible for diffuse ELP [9-11], but we were able to exclude them on the basis of the clinical history and autopsy findings. LAWLER [12] summarized the literature on 50 cases of "idiopathic cholesterol pneumonitis", however, they also differed from the present case because most of them were localized lesions and occurred in older patients. Congenital lipid storage disease was also unlikely, as the patient's serum lipid levels were normal and no abnormal lipid deposition was observed in any organ other than the lungs. Rheumatoid disease may be a possible aetiology, since his condition was tentatively diagnosed as juvenile rheumatoid arthritis at 3 yrs of age and rheumatoid and antinuclear factors were slightly elevated. But the patient had no evidence of rheumatoid disease, such as arthralgia or joint deformities, throughout his life. It is also possible that lung disease preceded the joint manifestation, however, the joint and lung symptoms usually develop within 5 yrs. Therefore, rheumatoid disease seems an unlikely aetiology of the lung disease in the present case.

Recent literature has indicated that the primary disease processes of coexisting ELP, PICG and PAP were seen in paediatric patients who have GOR with microaspiration $[4,13]$. The authors of these papers postulated that aspirated hydrochloric acid or foreign bodies (including exogenous lipids) could alter the metabolism of pulmonary surfactant, and result in abnormal layering and stacking of lipid bilayers in a variety of geometric arrangements. The pathological features they described resembled those in the present patient, who, however, differed from the previously reported cases as follows. Firstly, although neither barium swallow nor oesophageal $\mathrm{pH}$ probing could be carried out, he was unlikely to have had GOR in view of the clinical history and autopsy 
findings, in which the pathological changes were evenly distributed throughout the entire lung and neither anatomical abnormalities nor mucosal changes were found in the gastro-oesophageal junction. Secondly, all the previously reported cases had severe congenital disorders, such as cardiac anomalies, severe combined immunodeficiency (SCID), adenosine deaminase (ADA) deficiency, cystic fibrosis and severe neurological defects, whereas the present patient had no such background disease. Although it was not possible to determine the actual cause of the pathological processes responsible, this report may provide a new example of diffuse PICG and ELP coexisting with PAP in a patient with neither GOR nor serious background diseases.

It may be possible to speculate that the pathological process of this patient is similar to that involved in PAP, although the PAS-positive acellular material seen in PAP rarely causes inflammatory reaction and destructive changes [4, 14-16]. In other words, some unknown stimulus may facilitate type II pneumocytes to produce and/or fail to reabsorb surfactant material, or inhibit the clearance of alveolar lipid by alveolar macrophages. This hypothesis is supported by histological evolution observed in several patients with ELP coexisting with PAP, in which the predominant histological pattern changed from ELP to PAP (or vice versa) over a time interval of several years [4]. Whatever the mechanism of lipid accumulation in the alveolar and interstitial spaces, the lipid material may have elicited an inflammatory reaction, resulting in marked derangement of the native pulmonary structure.

In summary, we have reported a patient with diffuse pulmonary interstitial and intra-alveolar cholesterol granuloma and endogenous lipid pneumonia of unknown aetiology, which developed into respiratory failure over 15 yrs. This patient may provide a new example of diffuse-type pulmonary interstitial and intra-alveolar cholesterol granuloma and endogenous lipid pneumonia.

\section{References}

1. Dail DH. Bronchial and transbronchial diseases. In: Dail DH, Hammar SP, eds. Pulmonary Pathology. 2nd edn. New York, Springer-Verlag, 1994; pp. 79-119.
2. Spickard A, Hirschmann JV. Exogenous lipoid pneumonia. Arch Intern Med 1994; 154: 686-692.

3. Brown AC, Slocum PC, Putthoff SL, Wallace WE, Foresman BH. Exogenous lipoid pneumonia due to nasal application of petroleum jelly. Chest 1994: 105: 968969.

4. Fisher M, Roggli V, Merten D, Mulvihill D, Spock A. Coexisting endogenous lipoid pneumonia, cholesterol granulomas, and pulmonary alveolar proteinosis in a pediatric population: a clinical radiographic and pathologic correlation. Pediatr Pathol 1992; 12: 365-383.

5. Lipinski JK, Weisbrod GL, Sanders DE. Endogenous lipoid pneumonitis: pulmonary patterns. Am J Radiol 1981; 136: 931-934

6. Plas OV, Trigaux JP, Beers BV, Delaunois L, Sibille Y. Gravity-dependent infiltrates in a patient with lipoid pneumonia. Chest 1990; 98: 1253-1254.

7. Hugosson CO, Riff EJ, Moore CCM, Akhtar M, Tufenkeji HT. Lipoid pneumonia in infants: a radiographicalpathological study. Pediatr Radiol 1991; 21 193-197.

8. Glancy DL, Frazier PD, Robbert WC. Pulmonary parenchymal cholesterol-ester granulomas in patients with pulmonary hypertension. Am J Med 1968; 45: 198-199.

9. Costa-Jussa FR, Corrin B, Jacobs JM. Amiodarone lung toxity: a human and experimental study. J Pathol 1984; 143: 43-49.

10. Kay JM, Hasleton PS, Littler WA. Aetiology of pulmonary cholesterol-ester granulomas. Br J Dis Chest 1970; 64: 55-57.

11. Corrin B, King E. Experimental endogenous lipoid pneumonia and silicosis. J Pathol 1969; 97: 325-330.

12. Lawler W. Idiopathic cholesterol pneumonitis. Histopathology 1977; 1: 385-395.

13. McDonald JW, Roggli VL, Bradford WD. Coexisting endogenous and exogenous lipoid pneumonia and alveolar proteinosis in a patient with neurodevelopemental disease. Pediatr Pathol 1993; 14: 505-511.

14. Ramirez-R J, Harlan WR. Pulmonary alveolar proteinosis: nature and origin of alveolar lipid. Am J Med 1968; 45: 502-512.

15. Corrin B, King E. Pathogenesis of experimental pulmonary alveolar proteinosis. Thorax 1970; 25: 230-236.

16. Happleston AG, Fletcher K, Wyatt I. Change in the composition of lung lipids and the "turnover" of dipalpitoyl lecithin in experimental alveolar lipoproteinosis induced by inhaled quartz. Br J Exp Pathol 1974; 55: 384-395. 